\title{
THE LOW ENERGY PLASMA IN THE JOVIAN MAGNETOSPHERE
}

\author{
J. W. Belcher ${ }^{+}$, C. K. Goertz ${ }^{++}$, and H. S. Bridge * \\ ${ }^{+}$Department of Physics and Center for Space Research, MIT, Cambridge, Mass., 02139 \\ ${ }^{++}$Max-Planck-Institut für Aeronomie, Katlenburg-Lindau, West Germany
}

Abstract. Measurements below $6 \mathrm{keV}$ from the plasma science experiment on the Voyager spacecraft show that positive ions with temperatures as low as $30 \mathrm{eV}$ to several $\mathrm{keV}$ are observed to distances at least as great as $40 R_{\mathrm{J}}$ in the dayside Jovian magnetosphere. When velocity determinations are possible between $\sim 10 R_{\mathrm{J}}$ and $\sim 40 R_{\mathrm{J}}$, the plasma velocity component along the rigid corotation direction is found to be consistently less than the full corotation speed. Positive ion measurements above $\sim 28 \mathrm{keV}$ from the low energy charged particle experiment on Voyager demonstrate the existence of positive ions with temperatures of 20-30 keV at all distances greater than $30 R_{J}$. Taken together, these observations suggest that the low energy plasma population from $\sim 30 R_{J}$ to at least $40 R_{J}$ frequently contains both a cold and a hot component. A two-component plasma of this nature may indicate different sources, acceleration mechanisms, or time histories for the disparate components. It may also be indicative of a single acceleration mechanism which is highly energy dependent.

Introduction. Since the first in situ observations by Pioneers 10 and 11 , it has been conjectured that the Jovian magnetosphere contains hot plasma that significantly distorts the magnetic field from a dipolar shape (Smith et al., 1976). Since the major part of the plasma distribution was not measured directly, information about the bulk properties of the low energy Jovian plasma (i.e., composition, density, temperature and velocity) could only be obtained indirectly from theoretical reasoning based on the observed magnetic field topology (Goertz and Thomsen, 1979, and references therein).

The instrumentation on the Voyager spacecraft measures positive ion properties at much lower energies than did the Pioneer instruments. The plasma science experiment (PLS) covers the range from $10 \mathrm{eV}$ to $6 \mathrm{keV}$, and the low energy charged particle experiment (LECP) has a threshold for positive ions of $\sim 30 \mathrm{keV}$ for Voyager 1 and $\sim 28 \mathrm{keV}$ for Voyager 2 (Krimigis et al., 1979a,b). It was anticipated that this instrumentation would provide direct measurements of the bulk properties of the Jovian plasma. In the lo plasma torus, this is probably the case, since both by energy and number most of the positive ions appear to lie below $6 \mathrm{keV}$ (Bridge et al., 1979a; Sullivan and Bagenal, 1979; Bagenal et al., 1979). But, in other parts of the Jovian magnetosphere, the gap in the Voyager energy coverage, between $6 \mathrm{keV}$ and $28 \mathrm{keV}$, may be critical in terms of a complete description of the positive ion population which is important energetically. In particular on the basis of data taken outside of $30 R_{J}$ on Voyager 2, Krimigis et al., $1979 \mathrm{~b}$ conclude that most of the positive ions lie above $28 \mathrm{keV}$. These authors also argue that the positive ion distribution functions can be described (in the range from $28 \mathrm{keV}$ to $\sim 100 \mathrm{keV}$ ) as Maxwellians with temperatures of order $20-30 \mathrm{keV}$. Their reported number densities between 30 and $40 R_{J}$ range from 0.1 $\mathrm{cm}^{-3}$ to $5 \times 10^{-3} \mathrm{~cm}^{-3}$. For positive ions below $6 \mathrm{keV}$, the densities quoted by Bridge et al. $(1979 a, b)$ between 30 and $40 \mathrm{R}_{\mathrm{J}}$ range from $0.5 \mathrm{~cm}^{-3}$ to $5 \times 10^{-3} \mathrm{~cm}^{-3}$ for both Voyager 1 and 2 inbound. These PLS observations demonstrate that a $20-30 \mathrm{keV}$ Maxwellian is not a complete description of the total positive ion population at these distances. We elaborate on this point in the following sections and consider its implications in the discussion.

Characteristics of the Side-Looking Plasma Sensor. To explain the observations reported here, we must first describe the characteristics of the PLS instrument. The modulated grid Faraday cups on Voyager measure protons and electrons with energies from $10 \mathrm{eV}$ to $5950 \mathrm{eV}$; since the instrument measures energy per charge, the energy range for ions with charge number $Z^{*}$ is larger by a factor of $Z^{*}$.

Copyright 1980 by the American Geophysical Union
There are two energy resolutions available for measurements between $10 \mathrm{eV}$ and $6 \mathrm{keV}$ : the low resolution (L) mode, with 16 contiguous steps and $\triangle E / E \cong 29 \%$; and the high resolution (M) mode, with 128 contiguous steps and $\triangle \mathrm{E} / \mathrm{E} \cong 3.6 \%$. A detailed description can be found in Bridge et al. (1977). The instrument consists of four sensors, three of which (the A, B, and C sensors, or collectively, the main sensor) are arranged symmetrically about an axis which points toward the Earth, except during spacecraft yaw and pitch maneuvers. The fourth sensor, the D sensor, is directed at right angles to the symmetry axis of the main sensor. During most of the Voyager 1 and 2 inbound passes, the axis of the $D$ sensor points essentially into the expected corotation direction. It consequently measures significantly higher intensities than the main sensor during most of the two inbound passes. During the outbound passes, none of the detectors looked into the corotation direction when the spacecraft was in its nominal orientation. Our emphasis here will be on positive ion measurements in the D sensor on Voyager 1 and 2 inbound.

The electric currents measured by a Faraday cup are related to the ion distribution function in the following way (see Vasyliunas, 1971). Let $f(\vec{v})$ be the distribution function of an ionic species with mass number $A$ and charge number $Z^{*}, G(\vec{v}, n)$ the response function of a sensor whose axis lies along the unit vector $n$ (normalized to unity at normal incidence) and $A_{\text {eff }}$ the effective area at normal incidence. Suppose $\left\{\phi_{j}\right\}_{j=1}^{K+1}$ represents the set of contiguous voltages of the $\mathrm{K}$ energy channels, with $\phi$, the lower voltage of the $\mathrm{j}$-th channel. Let $e$ be the charge of the proton and $m_{p}$ its mass, and define the velocities $\left\{v_{j}\right\}_{j=1}^{K+1}$ by

$$
v_{j}=\left(2 \mathrm{eZ}^{*} \phi_{\mathrm{j}} / \mathrm{Am}_{\mathrm{p}}\right)^{1 / 2}
$$

We also define the average and difference velocities for the $j$-th channel by

$$
\bar{v}_{j}=\left(v_{j}+v_{j+1}\right) / 2 ; \Delta v_{j}=v_{j+1}-v_{j}
$$

Then the current $I_{j}$ in the $j$-th energy channel is

$$
I_{j}=Z * e A_{\text {eff }} \int_{v_{j}}^{v_{j}+1} v_{n} d v_{n} \int_{-\infty}^{\infty} \int_{-\infty}^{\infty} d v_{t 1} d v_{t 2} f(\vec{v}) G(\vec{v}, n)
$$

where $v_{n}=\vec{v} \cdot n$, and $v_{t 1}$ and $v_{12}$ are orthogonal velocity components tranverse to $n$

A good approximation to the $D$ sensor response function (Binsack, 1966; Sittler, 1978) is $\exp \left(-\alpha v_{t}{ }^{2} / v_{n}{ }^{2}\right)$, where $a$ is a constant value equal to 1.35 , and $v_{t}$ is $\left(v_{t 1}^{2}+v_{t 2}^{2}\right)^{1 / 2}$. Suppose we assume that $\mathrm{f}(\vec{v})$ is an isotropic convected Maxwellian with density $N$, thermal speed $W$ and bulk velocity $U$. If $\Delta v_{j} / W \ll 1$ (a good approximation for the magnetosphere plasma) and $\beta_{j}=\left(\bar{v}_{j} / W\right)^{2}$, then

$$
\begin{aligned}
& I_{j} \cong e Z^{*} A_{\text {eff }} \bar{v}_{j} \Delta v_{j}\left[\frac{N}{W} \operatorname{lvxp}\left(\frac{-\left(\bar{v}_{j}-U_{n}\right)^{2}}{W^{2}}\right)\right] \quad\left[\frac{\beta_{j}}{a+\beta_{j}} \exp \left(\frac{U_{t}{ }^{2} \alpha}{W^{2}\left(a+\beta_{j}\right.}\right)\right] \\
& \text { where } \quad U_{n}=\dot{U} \cdot n \text { and } U_{t}^{2}=U^{2}-U_{n}{ }^{2}
\end{aligned}
$$

For multiple species, we have the sum of terms similar to those shown in equation (4), one for each species.

The first factor in brackets in equation (4) is the reduced onedimensional distribution function along $\mathrm{n}$. The second factor in brackets contains effects due to the finite response of the cup. If $\alpha$ is identically zero (unit response for all directions of incidence), this factor is unity. For a high Mach number plasma $\left(U_{n} / W \gg 1\right)$ with flow almost directly into the $D$ sensor $\left(U_{t}^{2} / U_{n}^{2}<<\frac{1}{\alpha}\right)$, the second factor is close to unity when the first factor is appreciable. Under these conditions, $I_{j} /\left(\bar{v}_{j} \Delta v_{j}\right)$ is proportional to the reduced distribution 
function, and $\Sigma I_{j} / \bar{v}_{j}$ is proportional to the density $N$. This assumes that $U_{n}$ is less than $v_{K}$, the average velocity of our highest channel.

The currents measured in the Jovian magnetosphere are due to many ionic species. Frequently, these species are hot enough so that strong overlap occurs, and individual species cannot be identified. For this reason, the densities quoted by Bridge et al. $(1979 \mathrm{a}, \mathrm{b})$ are calculated from $\Sigma I_{j} /\left(e A_{e f f} \bar{v}_{j p}\right)$ where $\bar{v}_{j p}$ is the average velocity appropriate for protons. If the various ionic species are reasonably supersonic and within the energy range of PLS, then this number density is the weighted sum of the number densities of the various ions, with the weight for a given species equal to $\left(Z^{*}\right)^{3 / 2} / A^{1 / 2}$. This technique will yield density estimates which always underestimate the total number density even for supersonic flow within the PLS energy range (e.g., for pure $\mathrm{O}^{+}$, this density estimate will be too low by a factor of 4). Thus, the densities quoted by Bridge et al. $(1979 a, b)$ are too low by a factor of about 4 .

For future reference, consider the response of our detector when most of the positive ions lie above our maximum energy. For the heavy ion, $30 \mathrm{keV}$ Maxwellians reported by Krimigis et al. $(1979 b)$, both $U_{n}$ and $W$ are larger than $\bar{v}_{k}$, the average velocity of the highest PLS energy channel. In this case, for a single ionic species,

$$
\sum I_{j} /\left(e Z^{*} A_{e f f} \bar{v}_{j}\right) \lesssim N\left(\bar{v}_{k} / W\right)^{3}(3 \alpha \sqrt{\pi})^{-1}
$$

For Maxwellian distributions with these parameters, PLS would see little of the full density $\mathbf{N}$ because only a small fraction of the region in velocity space occupied by the positive ions is observed. In particular, for $30 \mathrm{keV}$ Maxwellians, the density estimate would be $\lesssim 1 \%$ of the hot plasma density $\mathrm{N}$ for singly ionized species, and $\leqslant 3 \%$ for doubly ionized species.

Departures from Rigid Corotation. We review briefly the nature of the spectra obtained by PLS on the inbound passes. Figure 1 shows a positive ion spectrum from the $D$ sensor taken near $20 R_{\mathrm{J}}$. We plot $I_{\mathrm{j}} /\left(\mathrm{e} A_{\text {eff }} \Delta \Phi_{\mathrm{j}}\right)$, where $\Delta \Phi_{\mathrm{j}}$ is the width of the energy channel in kilovolts. This quantity has units of \# $\left(\mathrm{cm}^{2} \mathrm{sec}\right.$ kilovolt) ${ }^{-1}$, and for a supersonic plasma is proportional to the reduced distribution function (cf. equation (4)). For such a cold spectrum, the various ionic species are well separated in energy per charge, and the densities, temperatures, and velocities into the $D$ sensor can be determined reasonably well for each species.

It is worth emphasizing that from this type of spectrum we can obtain what is essentially a model-independent determination of $U_{n}$, the velocity component along the axis of the sensor. The reasoning is as follows. The lowest peak in our energy per charge spectrum in Figure 1 must be due to protons. Other ionic species will appear at energies per charge of $A / Z^{*}$ times the proton energy per charge of $\sim 175$ volts. Peaks near $A / Z^{*}$ values of $8,10-2 / 3,16,23$, and 32 lead to plausible species identifications of $\mathrm{O}^{2+}, \mathrm{S}^{3+}, \mathrm{O}^{+}$or $\mathrm{S}^{2+}, \mathrm{Na}^{+}$, and $\mathrm{S}^{+}$, respectively (Sullivan and Bagenal, 1979). With these identifications we find that all of the species are moving into the $D$ sensor at a speed of $\sim 183 \mathrm{~km} / \mathrm{sec}$, as compared to the value of $\sim 238 \mathrm{~km} / \mathrm{sec}$ expected at this distance on the basis of rigid corotation (McNutt et al., 1979). Since a positive spacecraft charge would cause positive ions to be detected at less than their ambient energies, we could invoke a positive spacecraft potential to explain the observed departure from corotation. However, under this assumption it is impossible to assign reasonable values of $A / Z^{*}$ to the observed peaks.

Consider now the effects of the $D$ sensor response. It is easily shown from equation (4) that estimating $U_{n}$ by choosing the peak of the function $I_{j} /\left(e A_{\text {eff }} \Delta \Phi_{j}\right)$ will always overestimate the true value of $\mathrm{U}_{\mathrm{n}}$. For a reasonably supersonic flow, however, the fractional error in the estimate is proportional to $\alpha W^{4} / U_{n}^{4}+\alpha W^{2} U_{t}^{2} / U_{n}{ }^{4}$. The various species in Figure 1 are demonstrably supersonic, with thermal speeds of the order of $10 \mathrm{~km} / \mathrm{sec}$. Moreover, at the time of this observation, the $D$ sensor axis was within $7^{\circ}$ of the expected corotation flow direction and we do not expect the (undetermined) $U_{t}$ to be a significant fraction of $U_{n}$ at this distance $\left(\sim 20 R_{j}\right)$. Thus, the error in estimating $U_{n}$ by simply taking the peak is small. We actually determine our final plasma parameters for the various species in Figure 1 by least-squares model fits to Maxwellians. The point we emphasize is that for spectra with the features in Figure 1-i.e., those spectra allowing an unambiguous identification of the mean energies of three or more ionic species of high Mach number- our determination of the velocity component along the $D$ sensor axis is model-independent, with an accuracy of a fraction of a thermal width. To that accuracy, the estimate is independent of both our instrument characteristics and details of the distribution of the supersonic ions. When the plasma is warmer, both the response function of our D-cup and the details of the ion distribution functions become more important, and for that reason our velocity determinations become more model-dependent.

Between $10 R_{\mathrm{J}}$ and $22 R_{\mathrm{J}}$ on Voyager 1 inbound, there are numerous model-independent determinations of velocity components in the sense discussed above, because the plasma is distinctly cooler in this range than further out or further in (except for the extremely cold region near closest approach on Voyager 1). All of these determinations agree with the curve of McNutt et al. (1979). Further out on Voyager 1 inbound $\left(>22 \quad R_{\mathrm{J}}\right.$ ), resolved spectra become less numerous, reflecting an overall increase in the plasma temperature as we go out. Nonetheless, we do see resolved spectra in this region. Figure 2 shows data taken at $41 R_{\mathrm{J}}$. For a period of about 30 minutes around this time (day 621025 to 1052, Spacecraft Event Time), the spectra were cold enough for us to determine a velocity component into the D sensor of $\sim 204 \mathrm{~km} / \mathrm{sec}$ (as opposed to the expected 517 $\mathrm{km} / \mathrm{sec}$ for rigid corotation). Other cold spectra at $35 R_{\mathrm{J}}$ show a

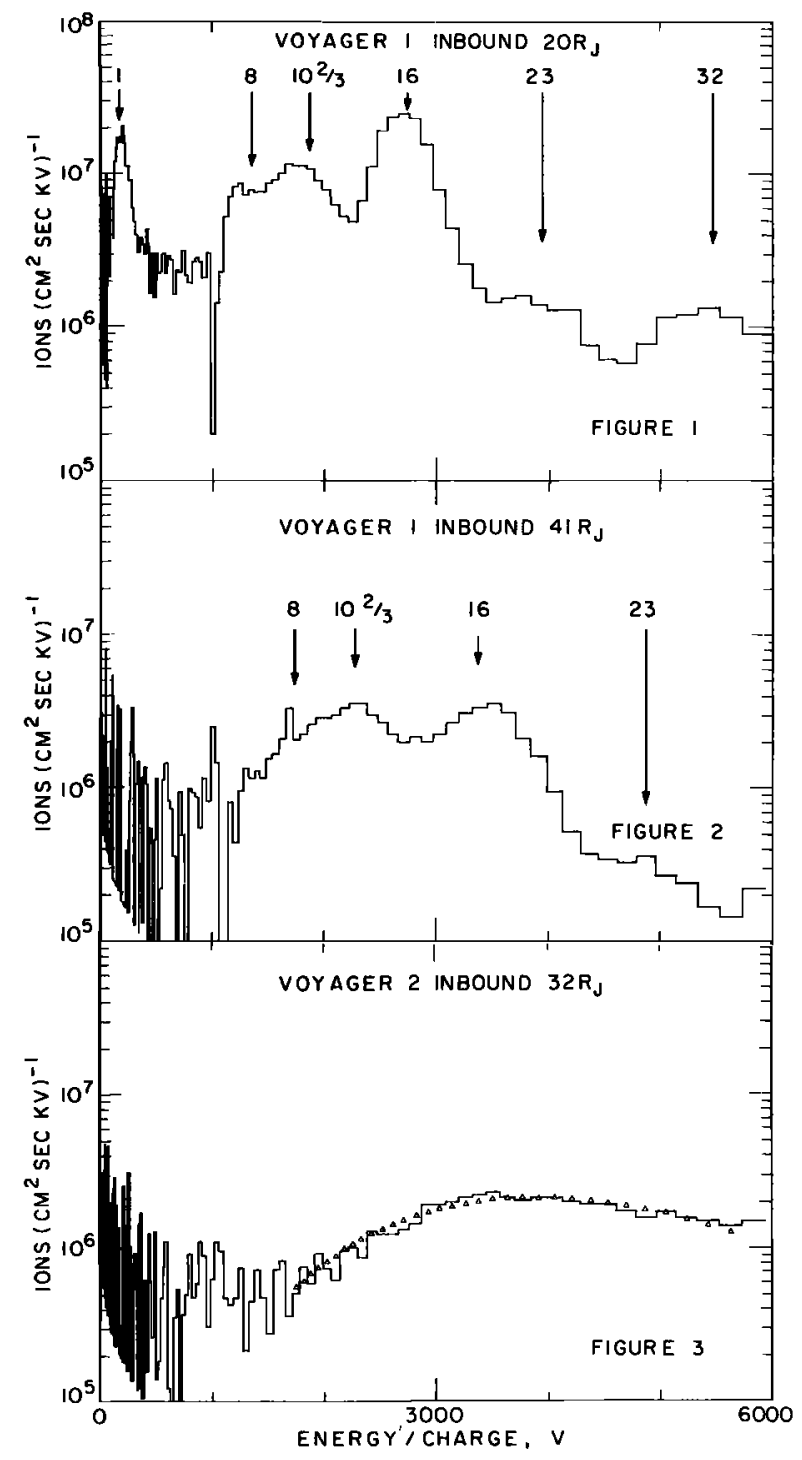

Figures 1-3: Positive ion "distribution functions" versus energy per charge (see text) 
similar departure from corotation $(212 \mathrm{~km} / \mathrm{sec}$ compared to an expected $437 \mathrm{~km} / \mathrm{sec}$ ). In both of these cases, the proton peak in the $M$ mode has disappeared into the noise. It is still seen in the $L$ mode however (the wider energy windows improve the signal to noise ratio), and its velocity is consistent with $\sim 200 \mathrm{~km} / \mathrm{sec}$ at these distances (cf. Figure 3 of Bridge et al., 1979a). The Voyager 2 data also show a lack of full corotation between $\sim 10 R_{I}$ and $\sim 20 R_{I}$ which is similar to that seen on Voyager 1. (see Figure 5 of Bridge et al., 1979b).

Positive Ion Properties Beyond $30 \mathbf{R}_{\mathbf{J}}$. In addition to estimates of $\mathrm{U}_{\mathrm{n}}$, we can also obtain estimates of the temperatures for the species shown in Figure 2. Maxwellian fits to the $\mathrm{S}^{2+}$ and $\mathrm{S}^{3+}$ peaks in Figure 2 yield a temperature of $\sim 30 \mathrm{eV}$ at $41 R_{\mathrm{J}}$. Fits to the $35 R_{\mathrm{J}}$ Voyager 1 inbound interval mentioned above yield similar temperatures. For spectra of this type, temperature estimates are quantitative and are based on few assumptions.

For Voyager 2 inbound, Bridge et al. (1979b), report that at a given radial distance the plasma is hotter than on Voyager 1. On Voyager 2 beyond $30 R_{J}$, we do not see the sort of resolved spectra found on Voyager 1 at these distances. More typical (and also typical of the hotter spectra on Voyager 1) is the spectrum in Figure 3, which occurs at $\sim 32 R_{\mathrm{I}}$ in a dayside plasma sheet crossing. It is, perhaps, worth noting specifically that the spectrum shown in Figure 3 cannot be explained in terms of background effects produced by isotropic energetic ions or hot electrons. If this were the case, signals larger than that seen in the D sensor would be observed in the main cluster (because the relative area solid angle product of the individual sensors in the main cluster is greater than that for the $\mathrm{D}$ sensor by about a factor of 3). In fact, the signal in the A, B, and C sensors is near the expected noise level and that in the $D$ sensor is $\sim 10$ times the noise level.

Our interpretation of this spectrum is that the various ionic species have become hot enough to smear out the individual peaks. For the dominant heavy ions observed by PLS in the Jovian magnetosphere, this will happen at relatively high Mach numbers. We lose resolution in the heavy ions when their Mach numbers drop below $\sim 4$, as is probably the case in Figure 3 . If we make the ad hoc assumption that we are seeing only $\mathrm{O}^{+}$in Figure 3, a single Maxwellian fit (as shown) yields a density of $0.3 \mathrm{~cm}^{-3}$, a thermal spread of $44 \mathrm{~km} / \mathrm{sec}$, and a velocity component $U_{n}$ of $220 \mathrm{~km} / \mathrm{sec}$. Clearly, our analysis is less quantitative here because of the overlap of the various hot ionic species. However, the temperature of order $200 \mathrm{eV}$ is probably an upper limit, for obvious reasons. Spectra taken near this time are hotter than the one shown in Figure 3, but the estimated temperatures are always less than several $\mathrm{keV}$.

Despite the less quantitative nature of this analysis, we do see large ion signals in our energy range at this time; the number densities below $6 \mathrm{keV}$ are of order $0.3 \mathrm{~cm}^{-3}$. For the hot Maxwellian plasma parameters quoted by Krimigis et al., $1979 \mathrm{~b}$ for $\mathrm{O}^{+}$at this time $(\mathrm{n}=$ $2.5 \times 10^{-2} \mathrm{~cm}^{-3}, \mathrm{U}=416 \mathrm{~km} / \mathrm{sec}, \mathrm{kT}=30 \mathrm{keV}$ ), we would expect to see ion intensities three orders of magnitude or more below our observed levels, corresponding to densities below $6 \mathrm{keV}$ of $\sim 10^{-4} \mathrm{~cm}^{-3}$ (cf. equations (4) and (5)). Thus, a $30 \mathrm{keV}$ Maxwellian at this density cannot be a complete description of the positive ion distribution at the time of this observation. The complete distribution function at this time must look qualitatively as shown in Figure 4-i.e., it consists of both a hot and cold component.

This two component nature of the plasma distribution function shown in Figure 4 must be fairly common outside of $\sim 30 R_{\mathrm{r}}$. For example, the densities given in Figure 3 of Bridge et al. (1979a) for Voyager 1 inbound are representative of Voyager 2 densities inbound. A comparison of this figure with Figure 7 of Krimigis et al., $1979 b$ shows that PLS densities are, in general, comparable to or much greater than LECP densities between $\sim 30$ and $\sim 40 R_{J}$. For example, on Voyager 2 at 0600 day 188 at $43 R_{J}$ inbound in a plasma sheet crossing the PLS lower limit assuming protons is $0.02 \mathrm{~cm}^{-3}$ or $0.08 \mathrm{~cm}^{-3}$ assuming $\mathrm{O}^{+}$. On the other hand, LECP "hot" Maxwellian densities at this time assuming oxygen are $0.01 \mathrm{~cm}^{-3}$. These values are representative of the seven plasma sheet crossings between $30 R_{J}$ and $43 R_{J}$ on Voyager 2 inbound.

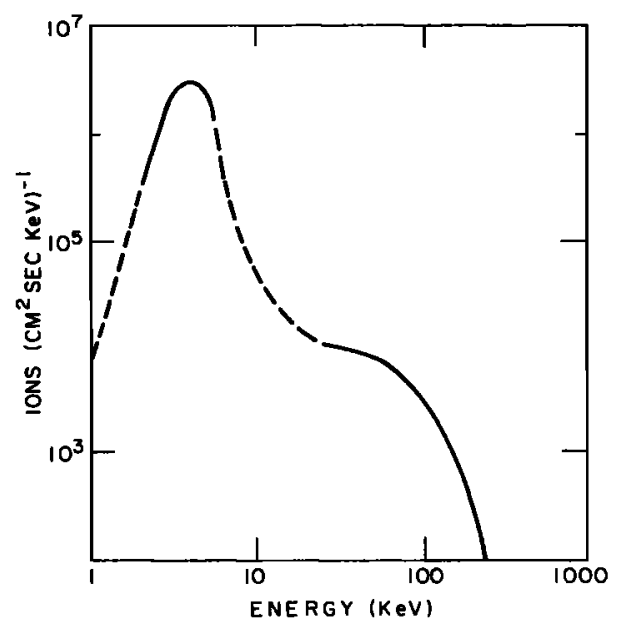

Figure 4: A sketch of the reduced one-dimensional distribution function at $\sim 32 R_{\mathrm{J}}$, assuming $\mathrm{O}^{+}$to be the dominant species.

Discussion. Knowledge of the properties of the Jovian magnetospheric plasma is essential for constructing realistic dynamical models of the magnetosphere, and for assessing the efficiency of the acceleration processes which must be active. We have compared the PLS and LECP positive ion measurements between $30 R_{J}$ and $40 R_{J}$, and find that in this region of the outer magnetosphere, the positive ions cannot be described by a single Maxwellian. Evidently, the plasma contains several components which may differ significantly in density and composition, and which differ drastically in temperature (20-30 $\mathrm{keV}$ as compared to several $\mathrm{keV}$ and less). By number density, the cold component may well be the dominant species, although by thermal energy density, the hot component may dominate. It may be that the relative importance of the cold and hot components in terms of thermal energy density is a strong function of local time and distance. Quantitative statements must await a detailed comparison of the PLS observations with LECP observations, as well as with results from the magnetic field and plasma wave investigations.

These results are of considerable theoretical interest. Hill $(1979 a, b)$ has interpreted the lack of full corotation as due to the effects of outward mass transport of heavy ions injected at lo. As we stated above, PLS sees some cooling of the Io torus ions in the region from $\sim 10 R_{\mathrm{J}}$ to $\sim 20 R_{\mathrm{J}}$, but beyond $20 R_{\mathrm{J}}$ the temperature increases with distance. Adiabatic cooling in the radially decreasing magnetic field would yield a relative change in energy proportional to the relative change in magnetic field. Since beyond $20 R_{J}$ the average energy of the ions does not change drastically with distance, a heating mechanism is required which must be at least strong enough to overcome the adiabatic cooling. From Io to the outer magnetosphere, the relative change in field is unity, so that the relative change in the energy is simply the initial energy at Io, which is $\sim 60 \mathrm{eV}$ (Bagenal et al., 1979). If we assume an injection rate of $10^{28}$ particles $\mathrm{sec}^{-1}$ (Hill, $1979 a, b)$, then the power required to overcome the adiabatic cooling of the ions is $10^{12} \mathrm{~W}$. This is a lower limit because the ion temperature actually increases with distance and because we expect additional losses of energy through ion-electron collisions, excitation of plasma waves, and so on.

The hot component also requires a heating mechanism, as argued by Krimigis et al. (1979b). Such a heating of energetic ions had been previously inferred on the basis of the Pioneer 10 and 11 measurements (Goertz and Thomsen, 1979, and references therein). Since the end product of these two heating mechanisms is so different (e.g., the temperatures of the two components are so different), the two components may experience different acceleration mechanisms, have different time histories, or stem from different sources. They may also be the result of a single acceleration mechanism which is highly energy dependent. Any of these possibilities imply that the nature of the particle acceleration and heating in the Jovian magnetosphere is a more complex process than previously suspected. 
Acknowledgments. We thank S. M. Krimigis for pointing out that an earlier version of this paper incorrectly quoted an LECP density estimate for $\mathrm{O}^{+}$at $32 \mathrm{R}_{\mathrm{J}}$ of $5 \times 10^{-3} \mathrm{~cm}^{-3}$; the published LECP density estimate is a factor of five larger. We also thank $F$. Bagenal for help in the derivation of plasma parameters for the various spectra and J. Sullivan, A. Lazarus, R. McNutt, S. Olbert, and V. M. Vasyliunas for useful comments. This work was supported in part by NASA contract 953733 with the Jet Propulsion Laboratory.

\section{References}

Bagenal, Fran, J. D. Sullivan and George L. Siscoe, Spatial distribution of plasma in the Io torus, Geophys. Res. Lett., this issue.

Binsack, Joseph H., Plasma studies with the IMP-2 satellite, pp 41-44, PhD thesis, MIT Cambridge, MA, August 1966.

Bridge, H. S., J. W. Belcher, R. J. Butler, A. J. Lazarus, A. M. Mavretic, J. D. Sullivan, G. L. Siscoe and V. M. Vasyliunas, The plasma experiment on the 1977 Voyager mission, Space Sci. Rev., 21, 259, 1977.

Bridge, H. S., J. W. Belcher, A. J. Lazarus, J. D. Sullivan, R. L. McNutt, F. Bagenal, J. D. Scudder, E. C. Sittler, G. L. Siscoe, V. M. Vasyliunas, C. K. Goertz and C. M. Yeates, Plasma observations near Jupiter: Initial results from Voyager 1, Science, 204, 987, 1979a.

Bridge, H. S., J. W. Belcher, A. J. Lazarus, J. D. Sullivan, R. L. McNutt, F. Bagenal, K. W. Ogilvie, J. D. Scudder, E. C. Sittler, V. M. Vasyliunas and C. K. Goertz, Plasma observations near Jupiter: Initial results from Voyager 2, Science, 206, 972, $1979 \mathrm{~b}$.

Goertz, C. K. and M. F. Thomsen, The dynamics of the Jovian magnetosphere, Rev. Geophys. Space Phys., 17, 731, 1979.
Hill, T. W., Inertial limit on corotation, J. Geophy. Res., 84, 6554, 1979a.

Hill, T. W., Corotational lag in Jupiter's magnetosphere: a comparison of observation and theory, Science, in press, 1979b.

Krimigis, S. M., T. P. Armstrong, W. I. Axford, C. O. Bostrom, C. Y. Fan, G. Gloeckler, L. J. Lanzerotti, E. P. Keath, R. D. Zwickl, J. F. Carbary and D. C. Hamilton, Low energy charged particle environment at Jupiter: a first look, Science, 204, 998, 1979a.

Krimigis, S. M., T. P. Armstrong, W. I. Axford, C. O. Bostrom, C. Y. Fan, G. Gloeckler, L. J. Lanzerotti, E. P. Keath, R. D. Zwickl, J. F. Carbary and D. C. Hamilton, Hot plasma environment at Jupiter: Voyager 2 results, Science, 206, 977, 1979 b.

McNutt, R. L., J. W. Belcher, J. D. Sullivan, F. Bagenal and H. S. Bridge, Departure from rigid corotation of plasma in Jupiters dayside magnetosphere, Nature, 280, 803, 1979.

Sittler, Edward C., Studies of the electron component of the solar wind and magnetospheric plasma, p 56, PhD thesis, MIT Cambridge, MA, February 1978.

Smith, E. J., L. Davis, Jr., and D. E. Jones, Jupiter's magnetic field and magnetosphere, in Jupiter, edited by T. Gehrels, University of Arizona Press, Tucson, 1976.

Sullivan, J. D. and F. Bagenal, In situ identification of various ionic species in Jupiter's magnetosphere, Nature, 280, 798, 1979.

Vasyliunas, V. M., in Methods of Experimental Physics, Vol. 9B of Plasma Physics, edited by R. H. Lovbergs, p. 49, Academic Press, 1971.

(Received November 5, 1979;

accepted November 21, 1979) 